\title{
Nonequilibrium Thermodynamics for Living Systems: Brownian Particle Description
}

\author{
Ulrich Zürcher \\ Physics Department, Cleveland State University \\ Cleveland, $\mathrm{OH} 44115$
}

USA

\section{Introduction}

In most introductory physics texts, a discussion on (human) food consumption centers around the available work. For example, the altitude is calculated a person can hike after eating a snack. This connection is natural at first glance: food is burned in a bomb calorimeter and its energy content is measured in "Calories," which is the unit of heat. We say that "we go to the gym to burn calories." This discussion implies that the human body acts as a sort of "heat engine," with food playing the role of 'fuel.'

We give two arguments to show that this view is flawed. First, the conversion of heat into work requires a heat engine that operates between two heat baths with different temperatures $T_{h}$ and $T_{c}<T_{h}$. The heat input $Q_{h}$ can be converted into work $W$ and heat output $Q_{c}<Q_{h}$ so that $Q_{h}=Q_{c}+W$ subject to the condition that entropy cannot be destroyed: $\Delta S=Q_{h} / T_{h}-$ $Q_{c} / T_{c}>0$. However, animals act like thermostats, with their body temperature kept at a constant value; e.g., $37^{\circ} \mathrm{C}$ for humans and $1-2^{\circ} \mathrm{C}$ higher for domestic cats and dogs. Second, the typical diet of an adult is roughly 2,000 Calories or about $8 \mathrm{MJ}$. If we assume that $25 \%$ of caloric intake is converted into useable work, a 100-kg adult would have to climb about 2,000 $\mathrm{m}$ [or approximately the height of Matterhorn in the Swiss Alps from its base] to convert daily food intake into potential energy. While this calculation is too simplistic, it illustrates that caloric intake through food consumption is enormous, compared to mechanical work done by humans [and other animals]. In particular, the discussion ignores heat production of the skin. At rest, the rate of heat production per unit area is $\mathcal{F} / A \simeq 45 \mathrm{~W} / \mathrm{m}^{2}$ (Guyton \& Hall 2005). Given that the surface area of a $1.8-\mathrm{m}$ tall man is about $A \simeq 2 \mathrm{~m}^{2}$, the rate of energy conversion at rest is approximately $90 \mathrm{~W}$. Since $1 \mathrm{~d} \simeq 9 \times 10^{4} \mathrm{~s}$, we find that the heat dissipated through the skin is $\mathcal{F} \simeq 8 \mathrm{MJ} / \mathrm{d}$, which approximately matches the daily intake of 'food calories.'

An entirely different focus of food consumption is emphasized in physiology texts. All living systems require the input of energy, whether it is in the form of food (for animals) or sun light (for plants). The chemical energy content of food is used to maintain concentration gradients of ions in the body, which is required for muscles to do useable work both inside and outside the body. Heat is the product of this energy transformation. That is, food intake is in the form of Gibbs free energy, i.e., work, and entropy is created in the form of heat and other waste products. In his classic text What is Life?, Schrödinger coined the expression that living systems "feed on negentropy" (Schrödinger 1967). Later, Morowitz explained that the steady state of living systems is maintained by a constant flow of energy: the input is highly organized energy [work], while the output is in the form of disorganized energy, and entropy 
is produced. Indeed, energy flow has been identified as one of the principles governing all complex systems (Schneider \& Sagan 2005).

As an example of the steady-state character of living systems with non-zero-gradients, we discuss the distribution of ions inside the axon and extracellular fluid. The ionic concentrations inside the axon $c_{i}$ and in the extracellular fluid $c_{o}$ are measured in units of millimoles per liter (Hobbie \& Roth 2007):

\begin{tabular}{|c||ccc|}
\hline Ion & $c_{i}$ & $c_{o}$ & $c_{o} / c_{i}$ \\
\hline $\mathrm{Na}^{+}$ & 15 & 145 & 9.7 \\
$\mathrm{~K}^{+}$ & 150 & 5 & 0.0033 \\
$\mathrm{Cl}^{-}$ & 9 & 125 & 13.9 \\
Misc. $^{-}$ & 150 & 30 & 0.19 \\
\hline
\end{tabular}

In thermal equilibrium, the concentration of ions across a cell membrane is determined by the Boltzmann-Nernst formula, $c_{i} / c_{o}=\exp \left[-z e\left(v_{i}-v_{0}\right) / k_{B} T\right]$, where $\Delta G=z e\left(v_{i}-v_{0}\right)$ is the Gibbs free energy for the potential between the inside and outside the cell, $\Delta v=v_{i}-v_{0}$. If the electrostatic potential in the extracellular fluid is chosen $v_{0}=0$, the 'resting' potential inside the axon is found $v_{i}=-70 \mathrm{mV}$. For $T=37^{\circ} \mathrm{C}$, this gives $c_{i} / c_{o}=13.7$ and $c_{i} / c_{o}=$ $1 / 13.7=0.073$ for univalent positive and negative ions, respectively. That is, the sodium concentration is too low inside the axon, while there are too many potassium ions inside it. The concentration of chlorine is approximately consistent with thermal equilibrium. Non-zero gradients of concentrations and other state variables are characteristic for systems that are not in thermal equilibrium (Berry et. al. 2002).

A discussion of living and complex systems within the framework of physics is difficult. It must include an explanation of what is meant by the phrase "biological systems are in nonequilibrium stationary states (NESS)." This is challenging, because there is not a unique definition of 'equilibrium state;' rather entirely different definitions are used to describe closed and open systems. For a closed system, the equilibrium state can be characrterized by a (multi-dimensional) coordinate $x_{\mathrm{s}}$, so that $x \neq x_{\mathrm{s}}$ describes a nonequilibrium state. However, the notion of "state of the system" is far from obvious for open systems. For a population model in ecology, equilibrium is described by the number of animals in each species. A nonequilibrium state involves populations that are changing with time, so a 'nonequilibrium stationary state' would correspond to dynamic state with constant (positive or negative) growth rates for species. Thus, any discussion of nonequilibrium thermodynamics for biological systems must involve an explanation of 'state' for complex systems. For many-body systems, the macroscopic behavior is an "emergent behavior;" the closest analogue of 'state' in physics might be the order parameter associated with a broken symmetry near a second-order phase transition.

This chapter is not a comprehensive overview of nonequilibrium thermodynamics, or the flow of energy as a mechanism of pattern formation in complex systems. We begin by directing the reader to some of the texts and papers that were useful in the preparation of this chapter. The text by de Groot and Mazur remains an authoritative source for nonequilibrium thermodynamics (de Groot \& Mazur 1962). Applications in biophysics are discussed in Ref. (Katchalsky \& Curram 1965). The text by Haynie is an excellent introduction to biological thermodynamics (Haynie 2001). The texts by Kubo and coworkers are an authoritative treatment of equilibrium and nonequilibrium statistical mechanics (Toda et al 1983; Kubo et al 1983). Stochastic processes are discussed in Refs. (Wax 1954; van Kampen 1981). Sethna gives a clear explanation of complexity and entropy 
(Sethna 2006). Cross and Greenside overview pattern formation in dissipative systems (Cross \& Greenside 2009); a non-technical introduction to pattern formation is found in Ref. (Ball 2009). The reader is directed to Refs. (Guyton \& Hall 2005) and (Nobel 1999) for background material on human and plant physiology. Some of the physics underlying human physiology is found in Refs. (Hobbie \& Roth 2007; Herman 2007).

The outline of this paper is as follows. We discuss the meaning of state and equilibrium for closed systems. We then discuss open systems, and introduce the concept of order parameter as the generalization of "coordinate" for closed systems. We use the motion of a Brownian particle to illustrate the two mechanisms, namely fluctuation and dissipation, how a system interacts with a much larger heat bath. We then briefly discuss the Rayleigh-Benard convection cell to illustrate the nonequilibrium stationary states in dissipative systems. This leads to our treatment of a charged object moving inside a viscous fluid. We discuss how the flow of energy through the system determines the stability of NESS. In particular, we show how the NESS becomes unstable through a seemingly small change in the energy dissipation. We conclude with a discussion of the key points and a general overview.

\section{Closed systems}

The notion of 'equilibrium' is introduced for mechanical systems, such as the familiar mass-block system. The mass $M$ slides on a horizontal surface, and is attached to a spring with constant $k$, cf. Fig. (1). We choose a coordinate such that $x_{\mathrm{eq}}=0$ when the spring force vanishes. The potential energy is then given by $U(x)=k x^{2} / 2$, so that the spring force is given by $F_{\mathrm{sp}}(x)=-d U / d x=-k x$. In Fig (1), the potential energy $U(x)$ is shown in black.

If the coordinate is constant, $x_{\mathrm{ns}}=$ const $\neq 0$, the spring-block system is in a nonequilibrium stationary state. Since $F_{\mathrm{sp}}=-d U /\left.d x\right|_{\mathrm{ns}} \neq 0$, an external force must be applied to maintain the system in a steady state: $F_{\text {net }}=F_{\mathrm{sp}}+F_{\text {ext }}=0$. If the object with mass $M$ also has an electric charge $q$, this external force can be realized by an external electric field $E, F_{\text {ext }}=q E$.

The external force can be derived from a potential energy $F_{\text {ext }}=-d U_{\text {ext }} / d x$ with $U_{\text {ext }}=-q E x$, and the spring-block system can be enlarged to include the electric field. Mathematically. this is expressed in terms of a total potential energy that incorporates the interaction with the electric field: $U \rightarrow U^{\prime}=U+U_{\text {ext }}$, where

$$
U^{\prime}(x)=\frac{1}{2} k x^{2}-F_{\mathrm{ext}} x=\frac{1}{2} k\left(x-x_{\mathrm{ns}}\right)^{2}-\frac{(q E)^{2}}{4 k} .
$$

The potential $U^{\prime}(x)$ is shown in red. That is, the nonequilibrium state for the potential $U(x)$, $x_{\mathrm{ns}}$ corresponds to the equilibrium state for the potential $U^{\prime}(x), x_{\mathrm{s}}^{\prime}$ :

$$
x_{\mathrm{ns}}=x_{\mathrm{s}}^{\prime}=\frac{q E}{k} .
$$

That is, the nonequilibrium stationary state for the spring-block system is the equilibrium state for the enlarged system. We conclude that for closed systems, the notion of equilibrium and nonequilibrium is more a matter of choice than a fundamental difference between them. For a closed system, the signature of stability is the oscillatory dynamics around the equilibrium state. Stability follows if the angular frequency $\omega$ is real:

$$
\omega^{2}=\frac{1}{M} \frac{d^{2} U}{d x^{2}}>0 \quad \text { (stability) }
$$


That is, stability requires that the potential energy is a convex function. Since $d^{2} U / d x^{2}=$ $d^{2} U / d x^{2}$, the stability of the system is not affected by the inclusion of the external electric force. On the other hand, if the angular frequency is imaginary $\omega=i \tilde{\omega}$, such that

$$
\frac{d^{2} U}{d x^{2}}<0 \quad \text { (instability) }
$$

The corresponding potential energy is shown in Fig. (2). The solution of the equation of motion describes exponential growth. That is, a small disturbance from the stationary state is amplified by the force that drives the system towards smaller values of the potential energy for all initial deviations from the stationary state $x=0$,

$$
x(t) \longrightarrow \pm \infty, \quad t \longrightarrow \infty ;
$$

the system is dynamically unstable. We conclude that a concave potential energy is the condition for instability in closed systems.

\section{Equilibrium thermodynamics}

Open systems exchange energy (and possibly volume and particles) with a heat bath at a fixed temperature $T$. The minimum energy principle applies to the internal energy of the system, rather than to the potential energy. This principle states that "the equilibrium value of any constrained external parameter is such as to minimize the energy for the given value of the total energy" (Callen 1960). A thermodynamic description is based on entropy, which is a concave function of (constrained) equilibrium states. In thermal equilibrium, the extensive parameters assume value, such that the entropy of the system is maximized. This statement is referred to as maximum entropy principle [MEP]. The stability of thermodynamic equilibrium follows from the concavity of the entropy, $d^{2} S<0$.

Thermodynamics describes average values, while fluctuations are described by equilibrium statistical mechanics. The distribution of the energy is given by the Boltzmann factor $p(E)=Z^{-1} \exp \left(-E / k_{B} T\right)$, where $Z=\int \exp \left(-E / k_{B} T\right) d E$ is the partition function. The equilibrium value of the energy of the system is equal to the average value, $E_{\mathrm{eq}}=\langle E\rangle=$ $\int d E p(E) E$. The fluctuations of the energy are $\delta E=E-\langle E\rangle$. The mean-square fluctuations can be written $\left\langle[\delta E]^{2}\right\rangle=k_{B} T^{2} \cdot d\langle E\rangle / d T$, or in terms of the inverse temperature $\beta=1 / T$, $\left\langle[\delta E]^{2}\right\rangle=-k_{B} d\langle E\rangle / d \beta$. Thus, the variance of energy fluctuations $\left\langle[\delta E]^{2}\right\rangle$ is proportional to the response of the systems $d\langle E\rangle / d \beta$. The proportionality between fluctuations and dissipation is determined by the Boltzmann constant $k_{B}=1.38 \times 10^{-23} \mathrm{~J} / \mathrm{K}$. Einstein discussed that "the absolute constant $k_{B}$ (therefore) determines the thermal stability of the system. The relationship just found is particularly interesting because it no longer contains any quantity that calls to mind the assumption underlying the theory" (Klein 1967).

In general, the state of an open system is described by an order parameter $\eta$. This concept is the generalization of coordinates used for closed systems, and was introduced by Landau to describe the properties of a system near a second-order phase transition (Landau \& Lifshitz 1959a). For the Ising spin model, for example, the order parameter is the average the average magnetization (Chaikin \& Lubensky 1995). In general, the choice of order parameter for a particular system is an "art" (Sethna 2006).

For simplicity, we assume a spatially homogenous system, so that $\eta(\vec{x})=$ const and there is no term involving the gradient $\nabla \eta$. The order parameter can be chosen such that $\eta=0$ in the symmetric phase. The thermodynamics of the system is defined by the Gibbs free energy 
$G=G(\eta)$. The equation of state follows from the expression for the external field $h=\partial G / \partial \eta$. The susceptibility $\chi=\partial\langle\eta\rangle / \partial h$ characterizes the response of the system. In the absence of an external magnetic field, the appearance of a non-zero value of the order parameter is referred to as spontaneous symmetry breaking (Chaikin \& Lubensky 1995; Forster 1975). The Gibbs free energy is written as a power series $G(\eta)=G_{0}+A \eta^{2}+B \eta^{4}$ with $B>0$. The symmetric phase $\eta=0$, corresponds to $A>0$, while $A<0$ in the asymmetric phase. The second-order coefficient vanishes at the transition point $A=0$. We only consider the case when the system is away from the transition point, so that $A \neq 0$, and write $\bar{\eta}=0$ and $\bar{\eta}= \pm A / 2 B$ for the respective minima of the Gibbs free energy, respectively. Since $\partial^{2} G /\left.\partial \eta^{2}\right|_{\eta=\bar{\eta}}=\chi^{-1}>0$, the susceptibility is finite and the variance of fluctuations of the order parameter is finite as well, with $\left\langle[\eta-\bar{\eta}]^{2}\right\rangle \sim \chi k_{B} T$, which is referred to as fluctuation-dissipation theorem [FDT].

A Brownian particle can be used to illustrate some aspects of equilibrium statistical mechanics (Forster 1975). In a microscopic description, a heavy particle with mass $M$ is immersed in a fluid of lighter particles of mass $m<M$. The time evolution is described by the Liouville operator for the entire system, and projection operator methods are used to eliminate the lighter particles' degrees of freedom [i.e., the heat bath]. It is shown that the interaction with a heat bath results in dissipation, described by a memory function and fluctuations characterized by stochastic forces. Because these two contributions have a common origin, it is not surprising that they are related to each other: the memory function is proportional to the autocorrelation function of the stochastic forces. The average kinetic energy of the heavy particle is given by the equipartition principle: $(M / 2)\left\langle v^{2}\right\rangle=k_{B} T / 2$. The memory function defines a correlation time $\zeta^{-1}$. For times $t>\zeta^{-1}$, a Langevin equation for the velocity of the heavy particle follows (Wax 1954). In one spatial dimension,

$$
\frac{\partial}{\partial t} v(t)+\zeta v(t)=\frac{1}{M} \zeta(t)
$$

We have the averages $\langle f(t)\rangle=0$ and $\langle f(t) v\rangle=0$. In Eq. (6), the stochastic force $\zeta$ is Gaussian "white noise:"

$$
\left\langle\zeta(t) \zeta\left(t^{\prime}\right)\right\rangle=2 \zeta M k_{B} T \delta\left(t-t^{\prime}\right) .
$$

The factor $2 \zeta M k_{B} T$ follows from the requirement that the stochastic process is "stationary." In fact, following Kubo, Eq. (7) is sometimes called the 'second fluctuation-dissipation theorem.' For long times, $t>>\zeta^{-1}$, the mean-square displacement increases diffusively:

$$
\left\langle[x(t)-x(0)]^{2}\right\rangle=\frac{2 k_{B} T}{M \zeta} t=2 D t .
$$

The expression for the diffusion constant $D=k_{B} T / M \zeta$ is the Einstein relation for Brownian motion, and is a version of the fluctuation-dissipation theorem. The diffusion constant can be written in terms of the velocity autocorrelation, $D=\int_{0}^{\infty} d t\langle\vec{v}(t) \vec{v}(0)\rangle$.

If the Brownian particle moves in a harmonic potential well, $U(x)=M \omega_{0}^{2} x^{2} / 2$, the Langevin equation is written as a system of two coupled first-order differential equations: $d x / d t=v$ and $d v / d t+\zeta v+\omega_{0}^{2} x=\zeta / M$. If the damping constant is large, the inertia of the particle can be ignored, so that the coordinate is described by the equation: $d x / d t+\left(\omega_{0}^{2} / \zeta\right) x=\zeta / M$. At zero temperature, the stochastic force vanishes, and the deterministic time evolution of the coordinate describes its relaxation towards the equilibrium $x=0: d x / d t=-\left(\omega_{0}^{2} / \zeta\right) x$ so that $x(t)=x_{0} \exp \left[-\left(\omega_{0}^{2} / \zeta\right) t\right]$.

In general, the relaxation of an initial nonoequilibrium state is governed by Onsager's regression hypothesis: the decay of an initial nonequilibrium state follows the same law as that 
of spontaneous fluctuations (Kubo et al 1983). The fluctuation-dissipation theorem implies that the time-dependence of equilibrium fluctuations is governed by the minimum entropy production principle. The stochastic nature of time-depedent equilibrium fluctuations is characterized by the conditional probability, or propagator $P\left(x, t \mid x_{0}, t_{0}\right)$. Onsager and Machlup showed that the conditional probability, or propagator, for diffusion can be written as a path integral (Hunt et. al. 1985):

$$
P\left(x, t \mid x_{0}, t_{0}\right)=\int \mathcal{D}[x(t)] \exp \left[-\frac{\zeta}{2 k_{B} T} \int_{t_{0}}^{t} K(s) d s\right],
$$

where $K(t)=(M / 2)(d x / d t)^{2}$ is the kinetic energy of the Brownian particle. The action $\int_{t_{0}}^{t} K(s) d s$ is minimized for the deterministic path (Feynman 1972). For fixed start $\left(x_{0}, t_{0}\right)$ and endpoints $(x, t)$, we find $K=(M / 2)\left[\left(x-x_{0}\right) /\left(t-t_{0}\right)\right]^{2}$. Gaussian fluctuations around the deterministic path yields: $P\left(x, t \mid x_{0}, t_{0}\right)=\left(4 \pi D\left[t-t_{0}\right]\right)^{-1 / 2} \exp \left[-\left(x-x_{0}\right)^{2} / 4 D\left(t-t_{0}\right)\right]$, which is the Greens function for the diffusion equation in one dimension $\partial P / \partial t=D \partial^{2} P / \partial x^{2}$ subject to the initial condtion $P\left(x, t_{0} \mid x_{0}, t_{0}\right)=\delta\left(x-x_{0}\right)$.

\section{Systems far from equilibrium}

We conclude that dissipation tends to 'dampen' the oscillatory motion around the equilibrium value $\bar{\eta}$, so that $\lim _{t \rightarrow \infty}\langle\eta(t)\rangle=\bar{\eta}$. Thus, a nonequilibrium stationary state $\eta_{\mathrm{s}} \neq \bar{\eta}$ requires the input of energy through work done on the system: highly-organized energy is destroyed, and dissipated energy is associated with the production of heat.

This mechanism is often illustrated by the Rayleigh-Benard convection cell, with a fluid being placed between two horizontal plates. If the two plates are at the same temperature, there is no macroscopic fluid flow, and the system is in the symmetric phase. An energy input is used to maintain a constant temperature difference across the plates. If $\Delta T$ is large enough, the component of the velocity along the vertical is non-zero, $v_{z} \neq 0$. A state with $v_{z} \neq 0$ is the asymmetric state of the fluid. Stationary patterns such as "stripes" and "hexagons" develop inside the fluid. Thus, the temperature difference $\Delta T$ can be viewed as the "force" maintaining stationary patterns in the fluid. Swift and Hohenberg showed that a potential $V(u)$ can be defined, such that the different stationary patterns correspond to local potential minima, cf. Fig (3). The dynamic of the system is first-order in time $\partial u / \partial t=-\delta V / \delta u$, where $\delta / \delta u$ is the functional derivative. If this energy flow stops, the velocity field in the fluid dissipates and the nonequilibrium patterns disappear.

A careful study of this system provides important insights into the behavior of nonequilibrium systems. Here, we are interested in systems for which nonequilibrium states are characterized by non-zero values of dynamic variables. A particularly simple model is discussed in Ref. (Taniguchi and Cohen 2008): a Brownian particle immersed in a viscous fluid moves at a constant velocity under the the influence of an electric force. The authors refer to it as, a Brownian particle immersed in a fluid "NESS model of class A." This model was used earlier by this author to illustrate nonequilibrium stationary states (Zurcher 2008). We note, however, that this model is not appropriate to discuss important topics in nonequilibrium thermodynamics, such as pattern formation in driven-diffusive systems. 


\section{Nonequilibrium stationary states: brownian particle model}

Our system is a particle with mass $M$ and the "state" of the system is characterized by the velocity $v$. The kinetic energy plays the role of Gibbs free energy:

$$
K(v)=\frac{M}{2} v^{2} .
$$

The particle at rest $v=0$ corresponds to the equilibrium state, while $v=$ const in a nonequilibrium state. We assume that the particle has an electric charge $q$, so that an external force is applied by an electric field $F_{\text {ext }}=q E$. Under the influence of this electric force, the (kinetic) energy of the particle would grow without bounds, $K(t) \rightarrow \infty$ for $t \rightarrow \infty$. The coupling to a 'thermostat' prevents this growth of energy. Here, we use a velocity-dependent force to describe the interaction with a thermostat. In the terminology of Ref. (Gallavotti \& Cohen 2004), our model describes a mechanical thermostat.

Dissipation is described by velocity-dependent forces, $f=f(v)$. For a particle immersed in a fluid, the force is linear in the velocity $f_{l}=6 \pi a \kappa v$ for viscous flow, while turbulent flow leads to quadratic dependence $f_{t}=C_{0} \pi \rho a^{2} v^{2} / 2$ for turbulent flow (Landau \& Lifshitz 1959b). Here, $\kappa$ is the dynamic viscosity, $\rho$ is the density of the fluid, and $a$ is the radius of the spherical object. These two mechanisms of dissipation are generally present at the same time; the Reynolds number determines which mechanism is dominant. It is defined as the ratio of inertial and viscous forces, i.e., $\operatorname{Re}=f_{t} / f_{l}=\rho a v / \kappa$. Laminar flow applies to slowly moving objects, i.e., small Reynolds numbers ( $\operatorname{Re}<1$ ), while turbulent flow dominates at high speeds, i.e., large Reynolds numbers $\left(\operatorname{Re}>10^{5}\right)$.

In the stationary state, the velocity is constant so that the net force on the particle vanishes, $F_{\text {net }}=q E-f=0$. We find for laminar flow,

$$
v_{\mathrm{s}}=\frac{q E}{6 \pi \kappa a}, \quad \operatorname{Re}<1,
$$

and for turbulent flow

$$
v_{\mathrm{S}}=\sqrt{\frac{q E}{C_{0} \pi \rho a^{2}}}, \quad \operatorname{Re}>10^{5} .
$$

In either case, we have $F_{\text {net }}>0$ for $v<v_{\mathrm{s}}$ and $F_{\text {net }}<0$ for $v>v_{\mathrm{s}}$; we conclude that the steady state is dynamically stable. These are, of course, elementary results discussed in introductory texts, where the nonequilibrium stationary state $v_{\mathrm{s}}$ is referred to as "terminal speed."

In general, the "forces" acting on a complex system are not known, so that the time evolution cannot be derived from a (partial-) differential equation. We will show how a discrete version of the equation can be derived from energy fluxes. To this end, we recall that in classical mechanics, velocity-dependent forces enter via the appropriate Rayleigh's dissipation function (Goldstein 1980). We deviate from the usual definition and define $\mathcal{F}$ as the negative value of the dissipation function such that $f=\partial \mathcal{F} / \partial v$, and $\mathcal{F}$ is associated with entropy production in the fluid. If the Lagrangian is not an explicit function of time, the total energy of the system $E$ decreases, $d E / d t=-\mathcal{F}$. For laminar flow, we have

$$
\mathcal{F}_{l}=3 \pi a \kappa v^{2} \quad(\text { laminar flow }),
$$

and for turbulent flow

$$
\mathcal{F}_{t}=\frac{C_{0} \pi}{3} \rho a^{2} v^{3}, \quad \text { (turbulent flow). }
$$


The Reynolds number can be expressed as a ratio of the dissipation functions, $\operatorname{Re} \sim \mathcal{F}_{t} / \mathcal{F}_{l}$. Since $\mathcal{F}_{l}>\mathcal{F}_{t}$ for $v \rightarrow 0$ and $\mathcal{F}_{t}>\mathcal{F}_{l}$ for $v \rightarrow \infty$, we conclude that the dominant mechanism for dissipation in the fluid maximizes the production of entropy.

The loss of energy through dissipation must be balanced by energy input in highly organized form, i.e., work for a Brownian particle. We write $d W=q E d x$ for the work done by the electric field, if the object moves the distance $d x$ parallel to the electric field. Since $v=d x / d t$, the energy input per unit time follows,

$$
\frac{d W}{d t}=\mathcal{W}=q E v
$$

We thus have for the rate of change of the kinetic energy,

$$
\frac{d K}{d t}=\mathcal{W}-\mathcal{F},
$$

cf. Ref. (Zurcher 2008). This is equivalent to Newton's second law for the object. In Fig. (5), we plot $\mathcal{F}$ (black) and $\mathcal{W}$ (in blue) as a function of the velocity $v$. The two curves intersect at $v_{\mathrm{S}}$, so that $\mathcal{F}=\mathcal{W}$, and the kinetic energy of the particle is constant $d K / d t=0$. We conclude that $v_{\mathrm{S}}$ corresponds to the nonequilibrium stationary state of the system, cf. Fig. (4).

The energy input exceeds the dissipated energy, $\mathcal{F}>\mathcal{W}$, for $0<v_{0}<v_{\mathrm{S}}$ so that the excess $\mathcal{W}-\mathcal{F}$ drives the system towards the stationary state $v_{0} \rightarrow v_{\mathrm{s}}$. For $v_{0}>v_{\mathrm{s}}$, the dissipated energy is higher than the input, $\mathcal{W}>\mathcal{F}$, so that the excess damping drives the system towards $v_{\mathrm{s}}$. That is, the nonequilibrium stationary state is stable,

$$
v_{0} \longrightarrow v_{\mathrm{s}}
$$

This result is independent of detailed properties of the open system. For $\partial^{2} W / \partial v^{2}=0$, the stability is a consequence of the convexity of the dissipative function

$$
\frac{d^{2} \mathcal{F}}{d v^{2}} \geq 0
$$

While a mechanical thermostat allows for a description of the system's time evolution in terms of forces, this is not possible for an open system in contact with an arbitrary thermostat. Indeed, a discrete version of the dynamics can be found from the energy fluxes $\mathcal{W}$ and $\mathcal{F}$. We assume that the particle moves at the initial velocity $0<v_{0}<v_{\mathrm{s}}$, so that $\mathcal{F}_{0}>\mathcal{W}_{0}$. We keep the energy input fixed, and increase the velocity until the dissipated energy matches the input $\mathcal{W}_{1}=\mathcal{F}_{0}$ at the new velocity $v_{1}>v_{0}$. This first iteration step is indicated by a horizontal arrow in Fig (5). The energy input is now at the higher value $\mathcal{W}_{1}>\mathcal{W}_{0}$, indicated by the vertical arrow. By construction, the inequality $\mathcal{W}_{1}>\mathcal{F}_{1}$ holds, so that the procedure can be repeated to find the the second iteration, $v_{2}$, cf. Fig. (5). A similar scheme applies for $v_{\mathrm{S}}<v_{0}<\infty$. We find the sequence $\left\{v_{i}\right\}_{i}$ for $i=0,1,2, \ldots$ with $v_{i+1}<v_{i}$ so that $\lim _{i \rightarrow \infty} v_{i}=v_{\mathrm{s}}$. Thus, for both $v_{0}<v_{\mathrm{S}}$ and $v_{0}>v_{\mathrm{S}}$, the initial state converges to the stationary state,

$$
v_{0} \longrightarrow v_{\mathrm{s}}
$$

We obtain a graphical representation of the dynamics by plotting the velocity $v_{i+1}$ versus $v_{i}$. This is sometimes called a cobweb or Verhulst plot (Otto \& Day 2007). The discrete version of the time evolution is indicated by the arrows, which shows that $v_{\mathrm{S}}$ is the fixed point of the time evolution, cf. Fig. (6). 
We generalize this result to the case when the dissipation function is concave,

$$
\frac{\partial^{2} \mathcal{F}}{\partial v^{2}}<0
$$

while keeping the behavior of the energy input fixed, i.e., $\partial^{2} W / \partial v^{2}=0$. We assume power law behavior for the dissipative function, so that concave behavior implies

$$
\mathcal{F} \sim v^{\alpha}, \quad 0<\alpha<1 .
$$

Since $f=\partial \mathcal{F} / \partial v \sim 1 / v^{1-\alpha}$, the velocity-dependent force diverges as the system slows down, i.e., $f \rightarrow \infty$ for $v \rightarrow 0$. The concave form of the dissipation function is unphysical for $v<v_{\mathcal{C}}$, where $v_{\mathcal{C}}$ is a cutoff. We ignore this cutoff in the following discussion. While this velocity-dependent function does not correspond to the behavior of any fluid, we retain the language appropriate for a Brownian particle. We now have the plot of the energy fluxes $\mathcal{F}$ and $\mathcal{W}$ as a function of velocity $v$, cf. Fig. (7).

The two curves intersect at the velocity $v_{\mathrm{S}}$ which characterizes the stationary state of the system. In this case, the dissipated energy exceeds the energy input for $0<v_{0}<v_{\mathrm{s}}$, so that the excess dissipation drives the system towards the equilibrium state $v=0$. For $v_{0}>v_{\mathrm{s}}$, the energy input is not balanced by the dissipated energy $\mathcal{W}>\mathcal{F}$. It follows that the excess input $\mathcal{W}-\mathcal{F}$ drives the state of the system away from the nonequilibrium stationary state.

We follow the same procedure as above, and assume that the initial velocity is (slightly) less than the stationary value, $0<v_{0}<v_{\mathrm{S}}$ so that $\mathcal{F}_{0}>\mathcal{W}_{0}$. We keep the energy input fixed, and decrease the velocity until $\mathcal{F}_{1}=\mathcal{W}_{0}$ at the velocity $v_{1}<v_{0}$. The iteration $v_{0} \rightarrow v_{1}$ is indicated by a horizontal arrow. We now have $\mathcal{F}_{1}>\mathcal{W}_{1}$, so that the steps can be repeated, cf. Fig. (6). In the case $v_{0}>v_{\mathrm{s}}$, we have $\mathcal{W}_{0}>\mathcal{F}_{0}$ so that the damping is not sufficient to act as a sink for the energy input into the system. Thus, the kinetic energy of the Brownian particle and the velocity increases, $v_{1}>v_{0}$. This step is indicated by a horizontal arrow. Since $\mathcal{W}_{1}>\mathcal{W}_{0}$, we find $\mathcal{W}_{1}>\mathcal{F}_{1}$, and the step can be repeated to find $v_{2}>v_{1}$. The corresponding phase portrait is shown in Fig. (7).

We conclude that the nonequilibrium stationary state $v_{\mathrm{S}}$ is unstable when the the dissipation function is concave. For $v_{0}<v_{\mathrm{s}}$, the initial state relaxes the towards the equilibrium state of the system,

$$
v_{0} \longrightarrow 0, v_{0}<v_{\mathrm{s}}
$$

while for $v_{0}>v_{\mathrm{s}}$, we find a runaway solution,

$$
v_{0} \longrightarrow \infty, \quad v_{0}>v_{\mathrm{s}}
$$

This instability is unique for nonequilibrium systems, and does not correspond to any behavior found for equilibrium systems. In fact, equilibrium thermodynamics excludes instabilities, because it is defined only for systems near local minima of the (free) energy. Exceptions are systems near a critical point, for which the free energy has a local maxima in the symmetric phase, and fluctuations diverge algebraically.

\section{Discussion}

A Brownian particle moving in a potential well can be used toexplain some aspects of equilibrium statistical physics. We used this model to explain certain aspects of nonequilibrium thermodynamics. A nonequilibrium stationary state corresponds to the 
particle moving at a constant velocity, under the influence of aexternal force. We also used this model to show how a NESS is sustained by a constant energy flow through the system. It is believedthat this is a key principle for steady states in open and complex systems (Schneider \& Sagan 2005); however, the behavior of open systems cannot be explained by the second law of thermodynamics alone (Farmer 2005; Callendar 2007).

We started from a heavy sphere immersed in a viscous fluid, so that in general, both viscous and laminar forces are acting on the sphere. Laminar flow applies to slowly moving spheres, whereas turbulent flow applies when spheres are moving fast. The crossover between linear and quadratic velocity-dependent forcesis based on the Reynolds number. We showed that this criterion coincides with maximum entropy production: laminar and turbulent flows are the dominant mechanisms for entropy production at small and large flow speeds, respectively. Ifa generalized version of Onsager's regression hypothesis holds for driven diffusive systems, the analysis of competing mechanismsfor entropy production may shed insight into the origin of the MEP principle. This principle was proposed as the generalization of Onsager's regression theorem to fluctuations in nonequilibrium systems (Martyushev \& Seleznev 2006; Niven 2009). MEP has beenused to explain complex behavior in ecology (Rhode 2005), earth science, and meteorology (Kleidon \& Lorenz 2005).

For the Brownian particle immersed in a fluid, the dissipation function is convex, $\partial^{2} \mathcal{F} / \partial v^{2}$, and the NESS is dynamically stable. That is, $v_{0} \rightarrow v_{\mathrm{s}}$ for arbitrary initial velocity. We generalized our discussion to more open systems, in which the particle velocity would correspond to a growth rate. We considered thecase whenentropy production is associated with a concave dissipation function $\partial^{2} \mathcal{F} / \partial v^{2}<0$, and found that the NESS is dynamically unstable:The system either relaxes towards the equilibrium statev $v_{0} \rightarrow 0$, or approaches a runaway solution, $v_{0} \rightarrow \infty$. The dynamics of an open system can be changed from stable to unstable by a variation in the dissipation function, or in the entropy production. Changes in metabolic rates have also been associated with disease (Macklem 2008): a decrease in the metabolic rate has been linked with a decrease in heart rate fluctuations in myocardial ischemia, while an increase in metabolic rate may be related to asthma.

It has also been proposed that the economy of a country or region can be considered an open system (Daley 1991), where an economy growing at a fixed rate, i.e., change of gross domestic power [GDP] per year would be in a nonequilibrium stationary state, or steady state. The population increase would correspond to an external force, while 'inefficiencies' such as wars, contribute to entropy production. If the analogue of a dissipation function for an economic system is concave, it might explain why monetary policies often fail to achieve stable growth of GDP over a sustained period. It would suggest that fluctuations of socio-economic variables are important since they can drive the system away from its steady state.

\section{References}

[Ball 2009] Ball, P. (2009). Nature's Patterns:A Tapestery in Three Parts (Oxford University Press, New York).

[Berry et. al. 2002] Berry, R. S.; Rice, S. A. \& Ross, J. (2002). Matter in Equilibrium:Statistical Mechanics and Thermodynamics (Oxford University Press, New York).

[Callen \& Welton 1951] Callen, H. B. \& Welton, T. A. (1951). Phys. Rev. 83, 34- 40.

[Callen 1960] Callen, H. B. (1960). Thermodynamics (J. Wiley \& Sons, New York).

[Callendar 2007] Callendar, C. (2007). Not so cool. Metascience 16, 147-151.

[Camazine et. al. 2001] Camazine, S.; Deneubourg, J.-L; Franks, N. R.; Sneyd, J.; Theraulaz, G. \& Bonabeau, E. (2001). Self-Organization in Biological Systems (Princeton University 
Press, Princeton, NJ).

[Chaikin \& Lubensky 1995] Chaikin, P. M. \& Lubensky, T. M. (1995). Principles of Condensed Matter Physics (Cambridge University Press, Cambridge, UK).

[Cross \& Greenside 2009] Cross M. \& Greenside, H. (2009). Pattern Formation and Dynamics in Nonequilibrium Systems. (Cambridge University Press, Cambridge, UK).

[Daley 1991] Dayley H. E. (1991). Steady-State Economics: Second Edition With New Essays (Island Press, Washington, DC).

[de Groot \& Mazur 1962] deGroot, S. R. \& Mazur, P. (1962). Non-Equilibrium Thermodynamics (North-Holland, Amsterdam).

[Farmer 2005] Farmer, J. D. (2005) Cool is not enough. Nature 436, 627-628.

[Feynman 1972] Feyman, R. P. (1972). Statistical Mechanics: A Set of Lectures (Benjamin Cummings,Reading, MA).

[Forster 1975] Forster, D. (1975). Hydrodynamic Fluctuations, Broken Symmetry, and Correlation Functions (Addison Wesley, Reading, MA).

[Gallavotti \& Cohen 2004] Gallavotti G. \& Cohen, E. G. D. (2004). Nonequilibrium stationary states and entropy. Phys. Rev. E 69035104.

[Goldstein 1980] Goldstein, H. (1980). Classical Mechanics, 2nd Ed. (Addison-Wesley, Reading, MA).

[Guyton \& Hall 2005] Guyton, A. C \& Hall. J. C. (2005) Textbook of Medical Physiology (Elsevier Saunder, Amsterdam).

[Haynie 2001] Haynie, D. T. (2001). Biological Thermodynamics (Cambridge University Press, New York).

[Herman 2007] Herman, I. P. (2007). Physics of the Human Body. (Springer-Verlag, New York).

[Hobbie \& Roth 2007] Hobbie, R. K. \& Roth, B. J. (2007). Intermediate Physics for Medicine and Biology 4th Ed. (Springer-Verlag, New York).

[Hunt et.al. 1985] Hunt, K. L. C.; Hunt, P. M. \& Ross, J. (1985). Path Integral Methods in Nonequilibrium Chemical Thermodynamics:Numerical Tests of the Onsager-Machlup-Laplace Approximation and Analytic Continuation Techniques. In Path Integrals from meV to MeV: Gutzwiller, M.C.; Inomata, A.; Klauder, J. R.; \& Streit, L. Ed. (World Scientific, Singapore).

[Katchalsky \& Curram 1965] Katchalsky, A. \& Curram, P. F. (1965). Nonequilibrium Thermodyanmics in Biology (Harvard University Press, Cambridge, MA).

[Kleidon \& Lorenz 2005] Kleidon, A. \& Lorenz, R. D. (eds.) (2005). Non-equilibrium Thermodynamics and the Production of Entropy: Life, Earth, and Beyond (Springer-Verlag, New York).

[Klein 1967] A. Einstein as quoted in Klein, M. J. (1967). Thermodynamics in Einstein's Thought. Science 157, 509-516.

[Kubo et al 1983] R. Kubo, M. Toda and N. Hashitsume (1983). Statistical Physics II:Nonequilibrium Statistical Mechanics (Springer-Verlag, New York).

[Landau \& Lifshitz 1959a] Landau, L. D. \&Lifshitz, E. M. (1959). Statistical Mechanics (Pergamon Press, London).

[Landau \& Lifshitz 1959b] Landau, L. D. \& Lifshitz, E. M. (1959). Hydrodynamics (Pergamon Press, London).

[Macklem 2008] Macklem P. (2008). Emergent Phenomena and the secrets of Life. J. App. Physiol. 104: 1844-1846.

[Martyushev \& Seleznev 2006] Martyushev, L. M. \& Seleznev, V. D. (2006). Maximum entropy production principle in physics, chemistry, and biology. Physics Reports 426,1-45. 
[Morowitz 1968] Morowitz, H. J. (1968). Energy flow in biology (Academic Press, New York).

[Nobel 1999] Nobel, P. S. Physicochemical \& Enviromnetal Plant Physiology 2nd Ed. (Academic Press, San Diego, CA).

[Niven 2009] Niven, R. K. (2009). Steady state of a dissipative flow-controlledsystem andthe maximum entropy production principle.Phys. Rev. E 80, 021113.

[Onsager 1931] Onsager, L. (1931). Reciprocal Relations in Irreversible Processes I, Phys. Rev. 37, 405-437; and Reciprocal Relations in Irreversible Processes II, Phys. Rev. 38, 2265 -2279.

[Otto \& Day 2007] Otto, S. P \& Day, T. (2007). A Bioloigist's Guide to Mathematical Modeling in Ecology and Evolution.(Princeton University Press, Princeton, NJ).

[Rhode 2005] Rhode R. (2005). Nonequilibrium Ecology (Cambridge University Press, Cambridge, UK).

[Schneider \& Sagan 2005] Schneider, E. D. \& Sagan, D. (2005). Into the Cold: Energy Flow, Thermodynamics and Life (The University of Chicago Press, Chicago).

[Schrödinger 1967] Schrödinger, E. (1967). What is Life with Mind and Matter \& Autobiographical sketches (Cambridge University Press, Cambridge UK).

[Sethna 2006] Sethna, J. P. (2006). Entropy, Order Parameters, and Complexity (Oxford University Press, New York).

[Taniguchi and Cohen 2008] Taniguchi, T. \& Cohen, E. G. D. (2008). Nonequilibrium Steady State Thermodynamics and Fluctuationsfor Stochastic Systems. J. Stat. Phys. 130, 633-667 (2008).

[Toda et al 1983] Toda, M.; Kubo. R.; \& Saito, N. (1983). Statitsical Physics 1: Equilibrium Statistical Mechanics (Springer-Verlag, New York).

[van Kampen 1981] Van Kampen, N. G. (183). Stochastic Processes in Physics and Chemistry (Elsevier, Amsterdam).

[Volkenstein 2009] Volkenstein, M. V. (2009). Entropy and Information (Birkhäuser Verlag, Basel, Switzerland).

[Wax 1954] Wax, N. Ed. (1954). Selected Papers on Noise and Stochastic Processes (Dover, New York).

[Zurcher 2008] Zurcher, U. (2008). Human Food Consumption: a primer on nonequilibrium thermodynamics for college physics. Eur. J. Phys. 29, 1183-1190.

\section{Figures}

(a)

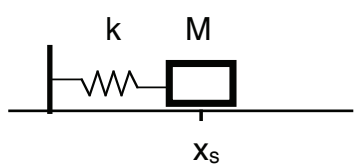

(b)

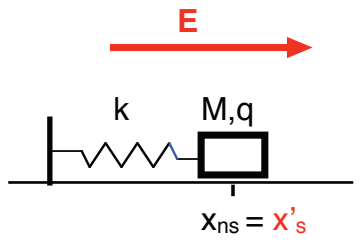

Fig. 1. The spring-block system (a) and with external force (b). 


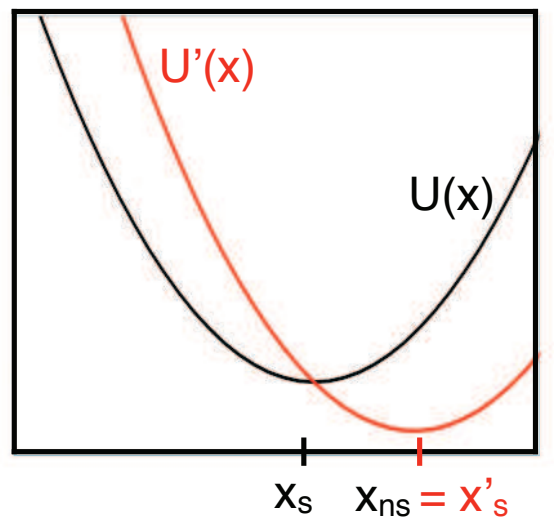

Fig. 2. The harmonic potential $U(x)=k x^{2} / 2$ [black] and the shifted potential $U^{\prime}(x)=U(x)-F_{\text {ext }} x$ [red].

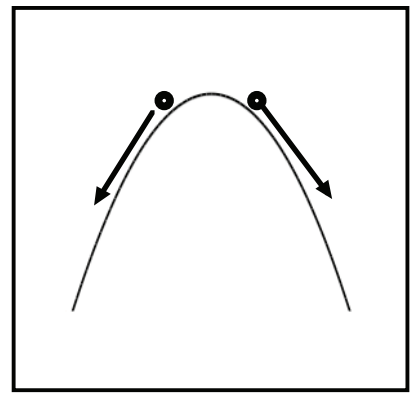

Fig. 3. The concave potential corresponding to a local maximum. 


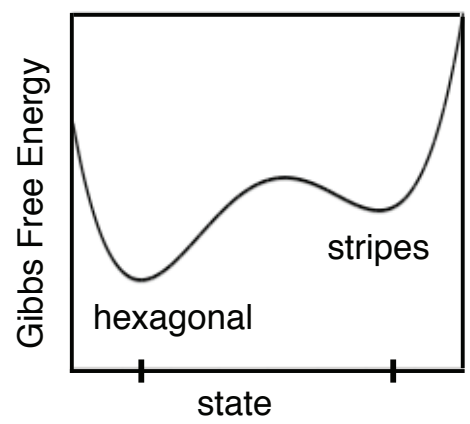

Fig. 4. The Gibbs free energy for the Rayleigh-Bernard connection cell.

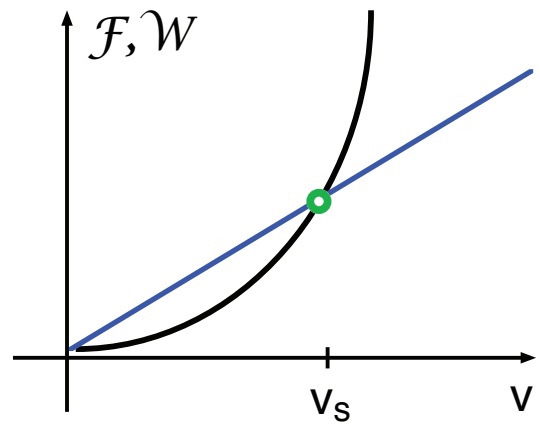

Fig. 5. The rate of energy input and energy dissipation for the Brownian particle immersed in a fluid. 


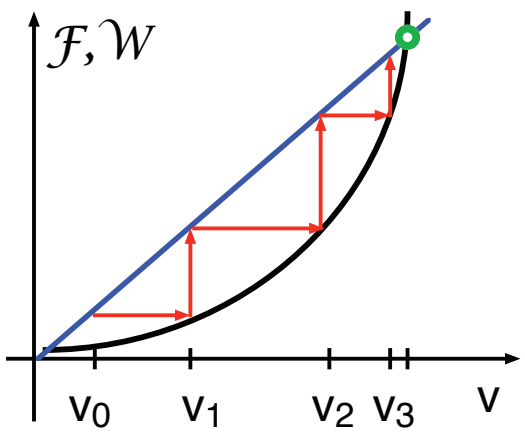

Fig. 6. Iterative time evolution for a Brownian particle with $v_{0}<v_{\mathrm{s}}$.

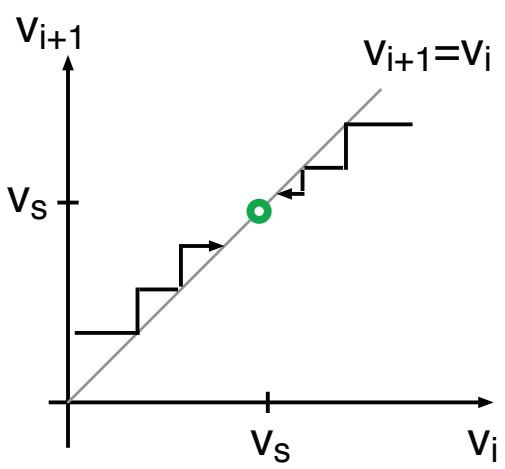

Fig. 7. The phase portrait for the discrete time evolution of the Brownian particle.

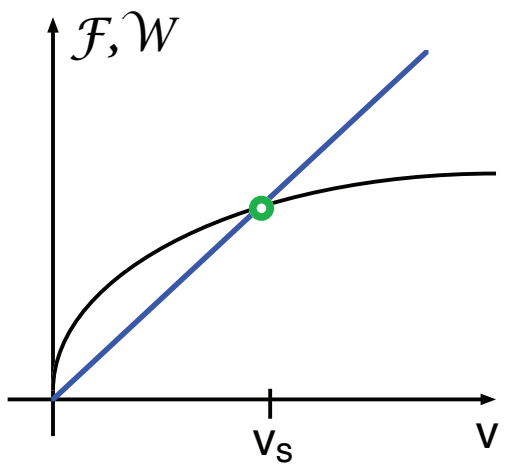

Fig. 8. The rate of energy input and energy dissipation for an open system with unstable dynamics. 


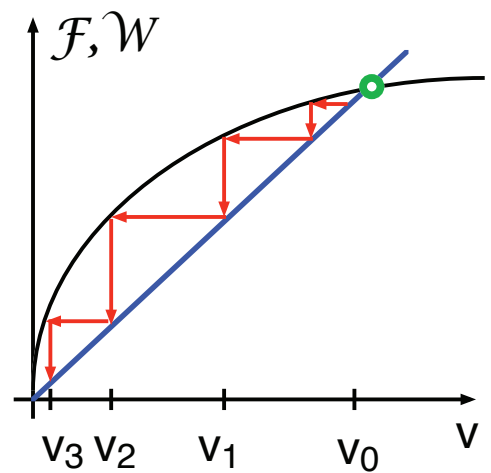

Fig. 9. Iterative time evolution for a unstable open system with $v_{0}<v_{\mathrm{s}}$.

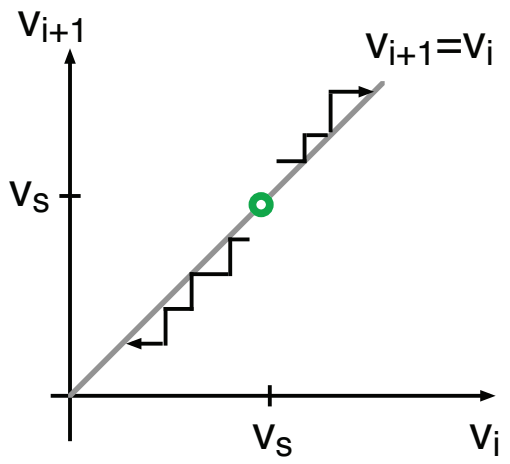

Fig. 10. The phase portrait for the discrete time evolution of an open system with unstable dynamics. 


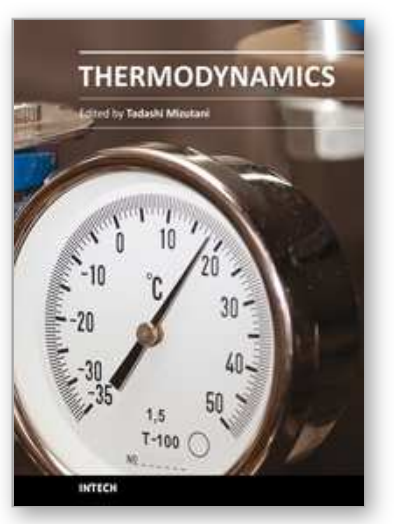

\author{
Thermodynamics \\ Edited by Prof. Mizutani Tadashi
}

ISBN 978-953-307-544-0

Hard cover, 440 pages

Publisher InTech

Published online 14, January, 2011

Published in print edition January, 2011

Progress of thermodynamics has been stimulated by the findings of a variety of fields of science and technology. The principles of thermodynamics are so general that the application is widespread to such fields as solid state physics, chemistry, biology, astronomical science, materials science, and chemical engineering. The contents of this book should be of help to many scientists and engineers.

\title{
How to reference
}

In order to correctly reference this scholarly work, feel free to copy and paste the following:

Ulrich Zurcher (2011). Nonequilibrium Thermodynamics for Living Systems: Brownian Particle Description, Thermodynamics, Prof. Mizutani Tadashi (Ed.), ISBN: 978-953-307-544-0, InTech, Available from: http://www.intechopen.com/books/thermodynamics/nonequilibrium-thermodynamics-for-living-systemsbrownian-particle-description

\section{INTECH}

open science | open minds

\author{
InTech Europe \\ University Campus STeP Ri \\ Slavka Krautzeka 83/A \\ 51000 Rijeka, Croatia \\ Phone: +385 (51) 770447 \\ Fax: +385 (51) 686166 \\ www.intechopen.com
}

\author{
InTech China \\ Unit 405, Office Block, Hotel Equatorial Shanghai \\ No.65, Yan An Road (West), Shanghai, 200040, China \\ 中国上海市延安西路65号上海国际贵都大饭店办公楼 405 单元 \\ Phone: +86-21-62489820 \\ Fax: $+86-21-62489821$
}


(C) 2011 The Author(s). Licensee IntechOpen. This chapter is distributed under the terms of the Creative Commons Attribution-NonCommercialShareAlike-3.0 License, which permits use, distribution and reproduction for non-commercial purposes, provided the original is properly cited and derivative works building on this content are distributed under the same license. 